\title{
Description of Temperature Dependence of Critical Micelle Concentration
}

\author{
Hong-Un Kim" and Kỵug-Hee Lim* \\ Department of Chemical Engineering, Chung-Ang Lniversitw, Seoul 156-756, Korea \\ Received January 29, 2003
}

\begin{abstract}
A new equation has been derived on the basis of $\Delta G^{\circ}=-R T \ln K$. linear behavior of the enthalpy of micellization with temperature. and the Gibbs-Helmholtz relation. It describes correctly the dependence of critical micelle concentration ( $\mathrm{K}_{\mathrm{CMC}}$ ) on temperature and has yielded excellent fitting results for various surfactant șistems. The new equation resılts in the linear behavior of the entropy of micellization with temperature and accounts for the compensation phenomena observed for the micellization in aqueous solutions. along with the linear dependence of the enthalpy of micellization on temperature. These results imply that the new equation of $\mathrm{Im}(T)$ accounts for the temperature dependence of $\mathrm{CMC}$ correctly
\end{abstract}

Key Words : Critical micelle concentration. Temperature dependence. Thermodynamic potentials of micellization

\section{Introduction}

For ionics and amphoterics. micellization is affected by temperature as the hydrophobic and head group interactions change with temperature. Accordingly. critical micelle concentration (CMC) versus temperature studies have been performed to obtain information on these interactions. ${ }^{l}$ For nonionic surfactants. CMC decreases with an increasing temperature due to an increase in hydrophobicity caused by the destruction of hydrogen bonds between water molecules and hydrophilic groups and the $\log \mathrm{CMC}$ is. $1 / T$ plot is nearly linear: Meanwhile, for ionic surfactants $\mathrm{CMC}$ decreases to a minimum value. characterized by the minimum CMC.

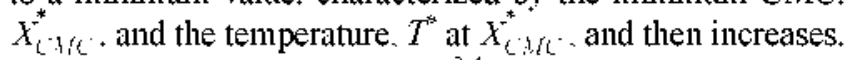
displaying a $U$-shaped behavior. ${ }^{3.4}$

When $\mathrm{CMC}$ is measured at various temperatures. the thermodynamic potentials of micellization can be determined. ${ }^{3-10}$ The standard Gibbs free energy of micellization per mole of surfactant or amphiphile. $\Delta G_{m, n}^{\prime}$. may be obtained from the relation $\Delta G_{i j:-i s}^{\prime \prime}=-R T \ln K / n$ with $K$ and $n$ being the equilibrium constant and association number. respectively, for the micellization. Then. the enthalpy of nicellization, $\Delta H_{m, z}^{\prime}$, is obtained from $\Delta G_{i n, z ;}^{\prime \prime}$ and the Gibbs-

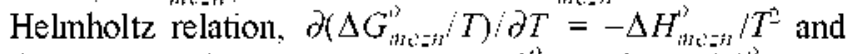
the entropy of the micellization, $\Delta S_{m, z}^{\prime}$. from $\Delta S_{i n, z ;}^{\prime \prime}=$

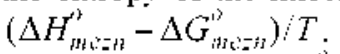

In determining $\Delta H_{m,-n}^{\prime}$ from $\Delta G_{i n:-3 ;}^{\prime}$ and the GibbsHelmholtz relation. the temperature dependence of $\Delta G_{i n:-z ;}^{\prime \prime}$ should be hlown so that its partial derivatives with respect to $T$ are calculated. This signifies that $X_{L 1,1<}$ should be described as a function of temperature and this has been done by expressing $X_{(1,1,}$ as polynomials of temperature. However. such description of $X_{C, k}(T)$ is not theory-based and therefore a theory-based and rigorous equation is

\footnotetext{
TPresent address: Department of Applied Chemistry, Dongyang Technical College, Seoul 152-714. Korea

${ }^{*}$ Corresponding Author. Tel: +82-2-820-5275, Fax: +82-2-8263574; E-mail: khlimatcauac.kr
}

desired.

In this article an equation of $X_{C \cdot M C}(T)$ is derived on the basis of $\Delta S_{n z=-1}^{i}=-R T \ln K / n$. linear behavior of $\Delta H_{m i=n}^{\prime \prime}$ $(T)^{\text {ll-l- }}$ and the Gibbs-Helmholtz relation. This equation has been also used in determination of $\Delta H_{m r_{i}:=t}^{\prime \prime}$ by Muller ${ }^{15}$ and $\mathrm{Singh}^{16}$ for specific surfactant systems. We have examined the equation for various surfactant syistems and the results are presented.

The equation fits the measured CMC data excellently for surfactant systems. It also accounts for the compensation phenomena. ${ }^{17-23}$ which states that $\Delta S_{m i=-17}^{i j}$ varies linearly with $\Delta H_{n i=1}^{i}$

\section{Gibbs Free Energy of Micellization and Critical Micelle Concentration (CMC)}

Thermodynamics of micellization has been often described by the models of mass action law and phase separation. and the former sheds more light on quantitative understanding of micellization. Let us consider the closed association model because it provides the essence of micellization without loss of generality. The model assumes that micelles comprised of $n$ surfactant molecules are found and that they are formed via the reaction:

$$
n S-m G=\left(S_{n} G_{n}\right)^{=}=M
$$

surfactant counterion micelle

in which $z$ is the charge or the valence of the micelles.

The standard Gibbs free energy change. $\Delta G^{\circ}$, for the micellization of eq. 1 can be obtained from the well-known thermodynamic result $\Delta G^{\circ}=-R T \ln K$ :

$$
\Delta G^{\circ}=-R T \ln K=-R T\left(\ln X_{M f}-n \ln X_{S}-m \ln X_{G}\right) .
$$

Here the equilibrium constant $K$ for the reaction is

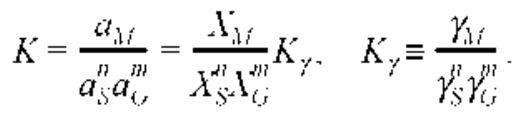

in which $a_{i}\left(=\gamma_{j} \mathrm{I}_{j}\right)$ is the activity of the species $i$ with $\gamma$ and 
$X$ being the activity coefficient and mole fraction. respectively, and $K_{y}=1$ is assumed. If we define $\Delta G_{m c=n}^{\prime \prime}$ as the standard Gibbs free energy change of micellization per mole of cmphiphile, i.e. $\Delta G_{m=n}^{o}=\Delta G^{\sigma} / n$. then

$$
\begin{aligned}
& \Delta G_{m: n}^{o}=\frac{1}{n} \mu_{H}^{\prime}-\mu_{S}^{\prime}-\frac{m}{n} \mu_{f ;}^{\prime} \\
& =-\frac{R T}{n} \ln K=-R T\left(\frac{1}{n} \ln X_{M,}-\ln X_{S}-\frac{m}{n} \ln X_{i j}\right)
\end{aligned}
$$

in which $\mu_{j}^{\prime \prime}$ is the standard chemical potential of species $i$.

Introducing the ratio $\sigma=X_{1 /} / X_{\mathrm{CMC}}$ as Tanford $\mathrm{did}^{-4}$ and recognizing that at the $\mathrm{CMC}, X_{S}=X_{C i C}-X_{\text {it }}$ and that $X_{i j} \approx X_{S}\left(=X_{(1, i c}\right)$ when the ionic surfactant $S_{v_{S}} G_{v_{i j}}$ is like a șimmetric electrolyte i.e. $v_{s}=v_{i j}$. we obtain

$$
\frac{\Delta G_{i n: ; n}^{\prime \prime}}{R T}=\left(1+\frac{m}{n}-\frac{1}{n}\right) \ln X_{L i,}-\frac{1}{n} \ln \sigma+\ln (1-\sigma) \text {. }
$$

The choice of $\sigma$ has only a small effect on the free energy of micellization because it ranges usually from 0.01 to 0.10 . Moreover for large $n$ (for example. $n \geq 50$ ) the third fourth. and fifth terms in the right-hand side are negligible. Hence. in this case eq. 5 is reduced to

$$
\frac{\Delta G_{m:-n}^{\prime \prime}}{R T}=(1+\beta) \ln X_{C, K C} .
$$

The fourth and fifth terms of eq. 5 may be elaborated ${ }^{22: 3}$ using the fact that physical properties of surfactant solutions change abruptly at the CMC. For a micellar system. the number of degrees of freedom is three. Accordingly the total surfactant concentration can determine the concentrations of every chemical species at constant temperature and pressure. On the basis of one mole of the total concentration the mass balances for counterions and surfactant ions yield

$$
\begin{gathered}
v_{j} X_{S t}=I_{i j}+n I_{i j} \\
v_{j} X_{S t}=X_{j}+m X_{i f}
\end{gathered}
$$

where $v_{i j}$ and $v_{f,}$ are the valences of the surfactant ion and counterion. respectively. of the surfactant $S_{1,} G_{1,}$ and $X_{i,}$ is the fraction of total surfactant. The CMC can be identified by the Philip's criterion ${ }^{25} d^{3} B d X_{S}^{* 3}=0$. Here $B$ is a property of the micellar solution. which may be represented by

$$
B=b_{S} X_{S}+b_{6 j} X_{i j}+b_{3,} X_{1\}}
$$

where $b_{3}$ is the contribution factor of each species and is related to the partial molar quantity of respective species.

After substantial mathematical manipulations ${ }^{26}$ for $d^{3} B / d X_{i ;}^{3}=0$, one obtains

$$
\begin{aligned}
& \frac{\Delta G_{i m n ; n}^{\prime \prime}}{R T}= \\
& {\left[\left(1+\frac{m}{n}-\frac{1}{n}\right) \ln X_{L: L C}\right]+\left[\frac{1}{n} \ln \frac{n(n+m)(2 n+2 m-1)}{n+m-2}\right]} \\
& +\left[\left(1+\frac{m}{n}-\frac{1}{n}\right) \ln \frac{1}{v_{S}} \frac{(n+m)(2 n+2 m-1)}{(n+m-1)(n+m+1)}\right]
\end{aligned}
$$

For $n>50$, the third term in the first bracket and the quantities in the second and third brackets become negligible and eq. 6 is recovered.

Eq. 10 is also employed for the micellization of nonionic amphiphiles, for which $m=0$ or $\beta=0$. Therefore it is reduced to

$$
\begin{aligned}
\frac{\Delta G_{n i=n}^{\prime}}{R T} & =\left[\left(1-\frac{1}{n}\right) \ln X_{(n / C}\right]\left[\frac{1}{n} \ln \frac{n^{2}(2 n-1)}{n-2}\right] \\
& +\left[\left(1-\frac{1}{n}\right) \ln \frac{1}{v_{S}} \frac{n(2 n-1)}{n^{2}-1}\right]
\end{aligned}
$$

Dropping $1 / n$ terms in eqs. 10 and 11 makes them reduced further to eqs. $12 \mathrm{a}$ and $12 \mathrm{~b}$.

$$
\begin{aligned}
& \frac{\Delta G_{m i n}^{i}}{R T}=\left(1+\frac{m}{n}\right) \ln X_{\text {(n) }}+\frac{1}{n} \ln 2 n(n+m) \\
& +\left(1+\frac{m}{n}\right) \ln \frac{2}{v_{i j}} \quad \text { (for ionics) } \\
& \frac{\Delta G_{n, i}^{i}}{R T}=\ln X_{C M}+\frac{1}{n} \ln 2 n^{2}+\ln \frac{2}{v_{\mathrm{S}}} \quad \text { (for nonionics) }
\end{aligned}
$$

For ionics eqs. 6 and $12 \mathrm{a}$ are good approximates to eq. 10 . Likewise. for nonionics eq. 6 with $\beta=0$ and eq. $12 \mathrm{~b}$ are good approximates to eq. 11. Details of mathematical derivation and comparison of terms in eq. 11 are found in reference 27 .

\section{Temperature Dependence of Heat of Micellization}

Heat of micellization or enthalpy of micellization. $\Delta H_{n n_{i}:-n}^{\prime \prime}$. has been determined in two ways: one is direct measurement with a calorimeter and the other is calculation from $\Delta G_{m i=n}^{i}$ and the Gibbs-Helnholtz relation.

Direct Method. It has been observed that experimentally measured $\Delta H_{n n_{i}, \lambda}^{\prime \prime}$ in aqueous surfactant solutions varies

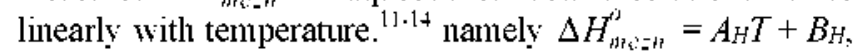
where $A_{H}$ and $B_{H}$ are the slope and intercept, respectively. in the $\Delta H_{m i=n}^{\prime}$ versus $T$ plot. Figure I shows such observations; Figure la for 8 liydrocarbon surfactants ${ }^{1]}$ and Figure lb for perfluoropolyether carboxylic acid and salt (H-. Na-PFPE) and perfluoroocatanoic acid and salt (H-. Na-PFO). ${ }^{12}$

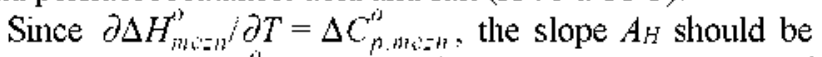
equivalent to $\Delta C_{n, m i n}^{i}$. the heat capacity change of micellization. The linearity between $\Delta H_{n i=-1}^{i j}$ and $T$ indicates that $\Delta C_{p, m i n}^{i}$ is constant at the observed temperature intervals and that. as shown in Figures 1 and 2. $\Delta C_{p, n i=n}^{\prime \prime}$ is positive for hydrocarbon surfactants and negative for perfluorinated ionic surfactants. If $\Delta H_{m, n}^{\prime}=0$ at the

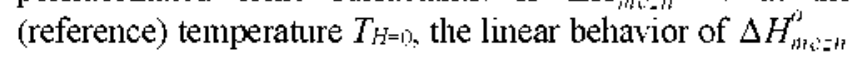
with $T$ can be stated as

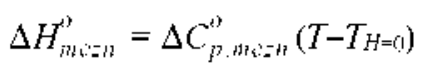

The heat capacity change. which is characteristic of the reactions in surfactant systems. is a consequence of solvation 

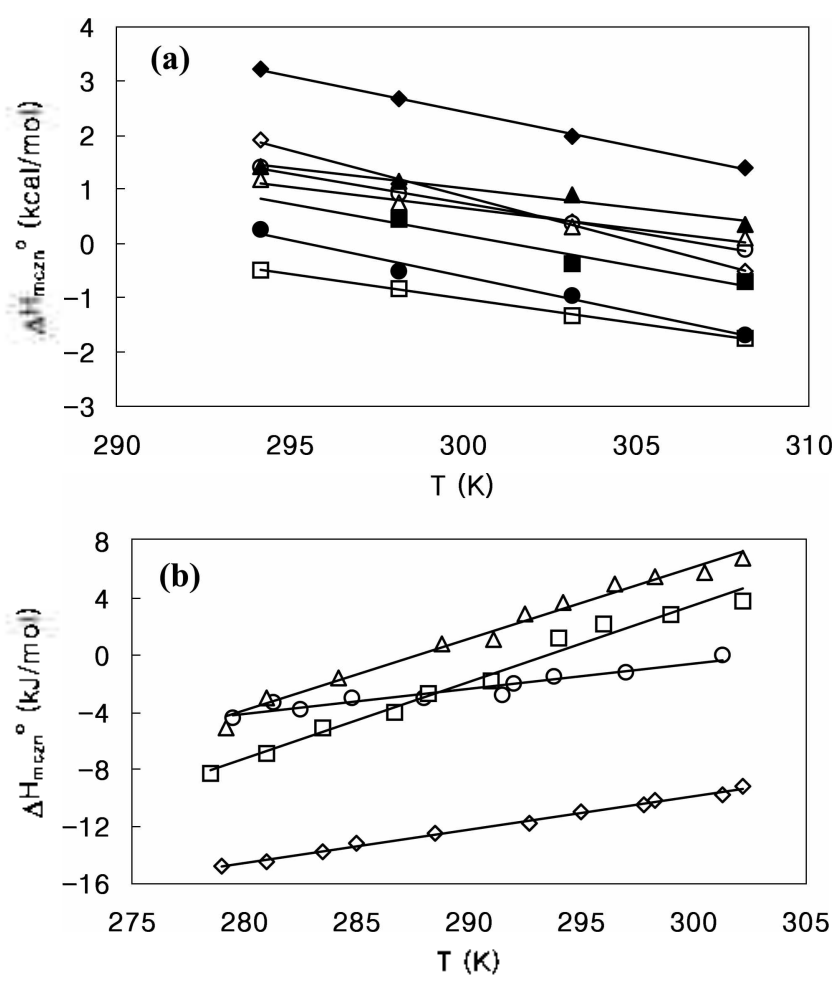

Figure 1. Calorimetric enthalpy change with temperalure of micelle formation in various aqueous surtactant solutions. (a) for dimethyldecylphosphine oxide $(\diamond)$. dimethyldodecylphosphine oxide $(, ;$ ) sodium dodecyl suldate $(\bullet)$. sodium dodecoyl sarcosinale (a). sodium octyl sulfate ( $\Delta$ ). sodium decyl sulfate ( - ). dodecylpyridinium bromide (a). dodecylpyridinium chloride $(\ulcorner$ ). and $(b)$ for perflorinated ionic surfactants. Na-PFPE (') ). H-PFPF. ( $)$ ). Na-PFO ( ᄀ) ). H-PFO ( $\therefore$ ).

and particularly of the structuring of water molecules in the vicinity of surfactant chains related to hydrophobicity, and is normally negative, ${ }^{28}$

Indirect Method. $\Delta I_{m c^{\prime \prime}=n}^{\prime \prime}$ may be determined from $\Delta G_{m(z)}^{\prime \prime}$ and the Gibbs-Helmholtz equation.

$$
\partial\left(\Delta G_{m c z \pi}^{\prime)} / T\right) / \partial T=-\Delta H_{m c=n}^{\prime \prime} / T^{2} .
$$

Since dropping lin terms would cause the error within $0.5 \%{ }^{27}$ eq. $12 \mathrm{a}$ can be used as a good approximate for $\Delta G_{m i z l}^{\prime \prime}$. Then one obtains

$$
\begin{aligned}
& \frac{\Delta H_{m i-s t}^{\prime \prime}}{R}=-T^{2} \frac{\partial}{\partial T}\left(\frac{\Delta G_{m c^{\prime}-t t}^{\prime \prime}}{R T}\right) \\
& -(1 \mid \beta) \frac{\partial}{\partial T} \ln X_{\text {(nH }} \cdot \ln X_{(H)} \frac{\partial}{\partial T}(1 \cdot \beta) \cdot \frac{\partial}{\partial \eta} f(n ; \beta) \frac{\partial n}{\partial T} \\
& =(1 \quad \beta) \frac{\partial}{\partial T} \ln X_{t}, h \cdot \frac{\partial f(n, \beta)}{\partial n} \frac{\partial n}{\partial T}
\end{aligned}
$$

where $f(n ; \beta)$ is defined as

$$
\begin{aligned}
f(n ; \beta) & -\frac{1}{n} \ln 2 n(n+m)+\left(1 \cdot \frac{m}{n}\right) \ln \frac{2}{v_{s}} \\
& =\frac{1}{n} \ln 2 n^{2}(1+\beta)+(1+\beta) \ln \frac{2}{v_{k}}
\end{aligned}
$$

The quantity $f(n ; \beta)$ is, in principle, a function of $n$ and $\beta(\equiv m / n)$. However, $\beta$, the degree of counterion binding, is weakly varying, although oscillatory in some cases. ${ }^{10,29}$ or almost constant with temperature. Hence, $f(n ; \beta)$ may be considered as a function of $n$ only and this is reflected in eq. 15.

Determination of $\Delta I I_{m c=1}^{\prime \prime}$ by the indirect method has been done usually by using eq. 15 without second term, i.e. neglecting the contribution by the second term $[\partial f(n ; \beta) / \partial n]$ $[\partial n / \partial T]$. Some researchers ${ }^{30.31}$ have asserted that such calculation of $\Delta I I_{m<\Omega /}^{\prime \prime}$ is not proper because of the large changes in asgregation number that can occur with temperature. However, Krescheck ${ }^{11}$ and Kiraly ${ }^{13}$ have defended this procedure, chiefly on the ground that the $\Delta I I_{m(z)}^{\prime \prime}$ thus calculated agree with the calorimetric estimates.

\section{Temperature Dependence of Critical Micelle Concentration}

A new description of the thermal behavior of critical micelle concentration is derived from $\Delta G^{\prime}=-R T \ln K$, the linear behavior of $\Delta I_{m i=n}^{*}(T)$, and the Gibbs-Helmholtz. relation, $i$.e, from egs, $12 \mathrm{a}, 13$, and 14. From the last two equations one obtains

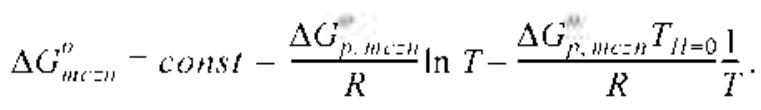

Combining eqs. $12 \mathrm{a}$ and 22 now yields

$$
\ln X_{(x H}=A+B \ln T+\frac{C}{T}-\frac{1}{(1+\beta) n} \ln 2 n^{2}(1-\beta)-\ln \frac{2}{v_{i}}
$$

where $A$ is a undetermined coefficient, and $B$ and $C$ are defined as

$$
\begin{aligned}
& B \equiv \frac{-\Delta G_{p .}^{\prime \prime}}{(I-\beta) R}>0 \\
& C=\frac{-\Delta G_{H, H C=n}^{\prime \prime} T_{I I-0}}{(1+\beta) R}>0
\end{aligned}
$$

$B$ and $C^{*}$ are positive, since $\Delta G_{p, m c m}^{\prime \prime}$ is negative. $T_{H-0}$ is around a room temperature.

The fourth and the fifth terms of eq. 18 is negligibly small compared to the first three terms and therefore they can be dropped to obtain

$$
\ln Y_{C: H C}=A+B \ln T+\frac{C}{T}
$$

\section{Results and Discussion}

Because eq. 20 has three fitting parameters like a $2^{\text {nd }}$-order polynomial. fits by both equations are examined. Figure 2 shows the results of the fits by these cquations for dodecyl-4methoxypyridinium chloride. Both equations lit the data well and eq. 20 fits slightly better than the $2^{\text {nd }}$-order 


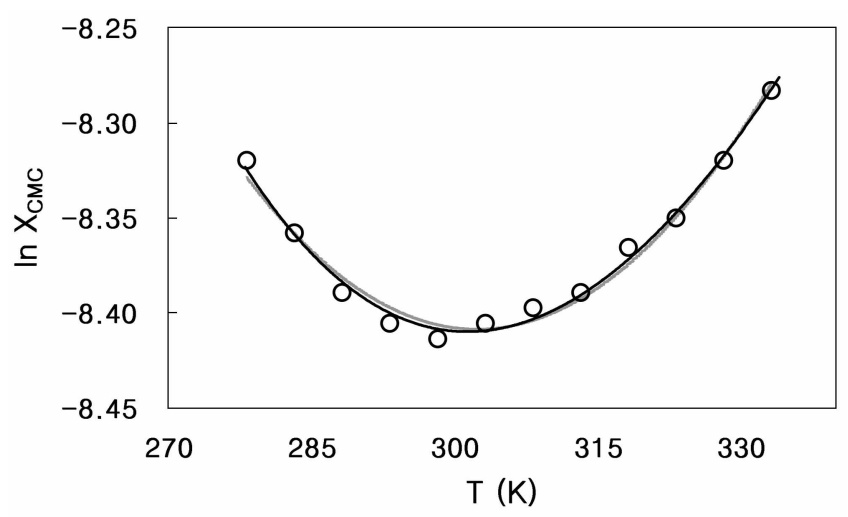

Figure 2. Comparison of $2^{\text {rul }}$-order polynomial titting and our equation litting for dodecy)-4-methoxypyridinium chloride (gray line: $2^{\text {nd }}$ polynomial litting. full line: our functional form fitting).

polynomial (correlation coefficients, 0.9958 vs. 0.9883).

Fa. 20 has been employed to fit CMC data of various surfactant systems and it yields the results which are in excellent agreement with the measured data. Figures 3-6 show the results for nonionic n-dodecyl polyethylene glycol monoether $\left(C_{12} E_{1}, C_{12} F_{6}, C_{12} E_{8}\right)^{8} \alpha$-sulfonatomyristic acid methyl ester, ethyl ester, ${ }^{2 z}$ cationic alkyltrimethylammonium

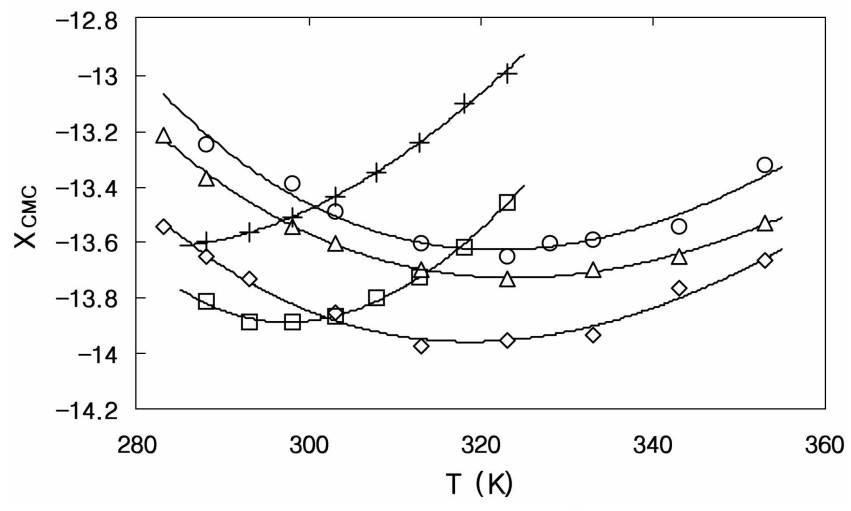

Figure 3. E. (20) litting of nonionic surfactant solutions $(\ulcorner$ :

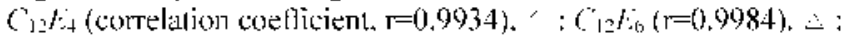
$C_{12} L_{8} \quad(1=0.9899)$. $\therefore \alpha$-sulfonatomyristic acid methyl ester $(\mathrm{r}=0.9980) .+: \alpha$-sulfonatomyristic acid ethy l ester $(\mathrm{r}=0.9994))$.

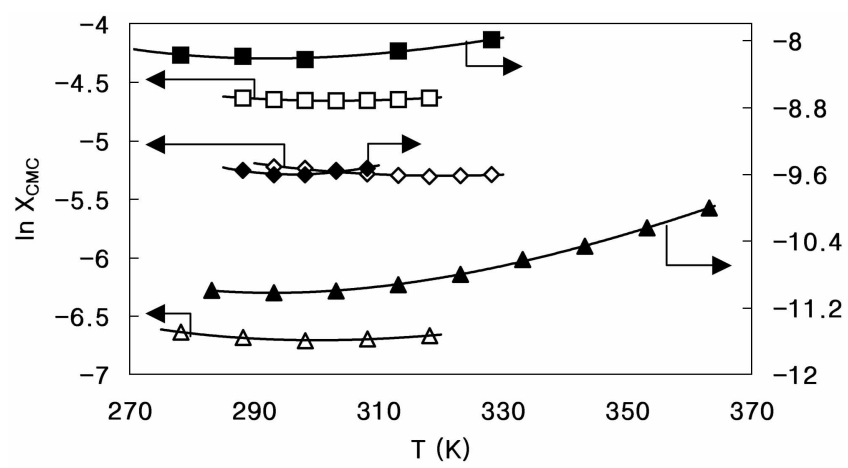

Figure 4. Lq. (20) fitting of cationic surfactant solutions ( $L: i=6$ (correlation coefficient. $\mathrm{r}=0.9989)$. $,: i=8(\mathrm{r}=0.9812) . \therefore: i=10$ (r 0.9924). $\square: i$ I2 (r 0.9882). : i 14 (r 0.9777). \& : i 16 $(\mathrm{r}=0.9996))$.

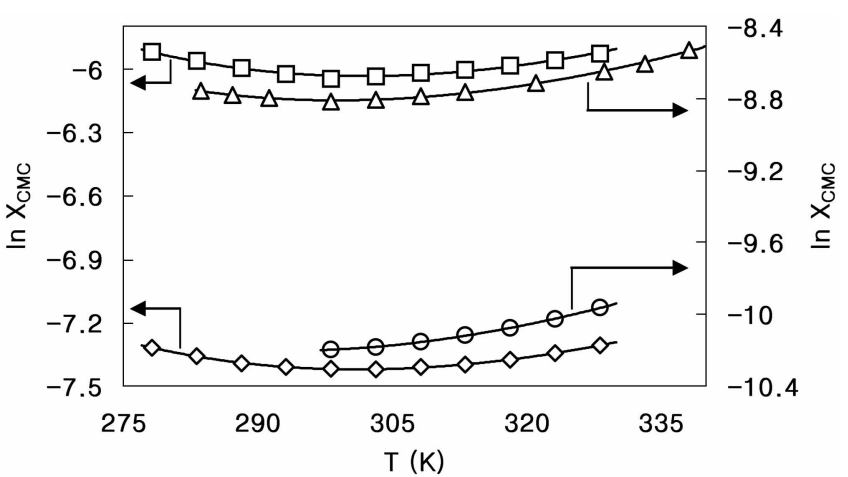

Figure 5. Lu. (20) fitting of anionic surfactant sodium alkyl (\lrcorner : octyl (correlation coefficient. $r=0.9866)$.' : decyl $(r=0.9992)$. $\therefore$ : dodecyl ( $\mathrm{r} 0.9984)$, : tetradecy ( $\mathrm{r} 0.9997)$ ) sultate solutions.

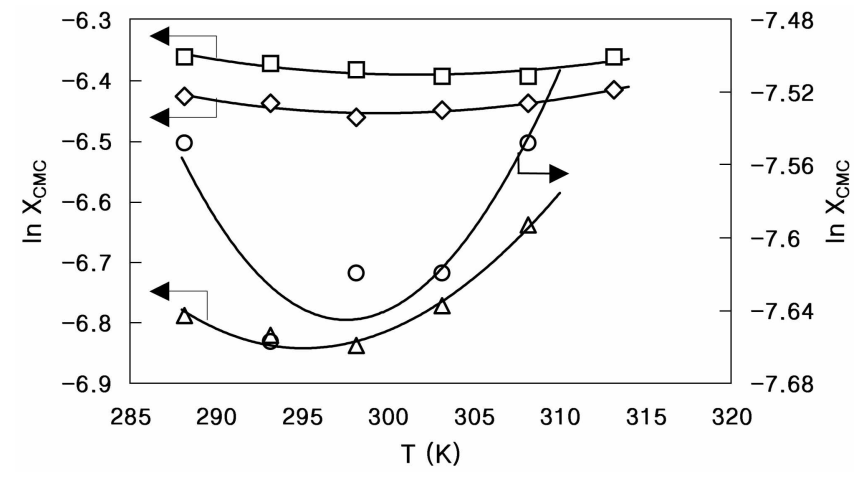

Figure 6. F.q. (20) litting of amphiphilic drugs ( 7 : cloxacillin (correlation coetlicient. $\mathrm{r}=0.9725) . \quad$ : dicloxacillin $(\mathrm{r}=0.9765)$. $\therefore$ : imipramine $(r=0.9913)$, : elomipramine $(r=0.9733))$ solutions.

bromide $\left(C, T A B, i=6,{ }^{32} 8,^{\circ} 10,312 .^{34} 14, r^{35} 16^{76}\right)$, anionic (sodium alkyl sulfate, SOS, SDeS, SDS, STS ${ }^{3.378}$ ) surfactants, and amphiphilic drugs (cloxacillin, dicloxacillin, ${ }^{39}$ imipramine, clomipramine ${ }^{40}$ ), respectively. The results in these figures imply that eq. 20 describes correctly the temperature dependence of CMC for most of surfactant systems.

The entropy change, $\Delta S_{m i z n}^{\prime}$, for the micellization can be obtained from the relation $T \Delta S_{m, 7 n}^{\prime \prime}=\Delta H_{m, \cdots n}^{\prime \prime}-\Delta G_{m, n+m}^{\prime \prime}$. Hence, one obtains

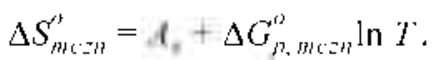

This equation exhibits the overall behavior as shown in rigure 7. However, the linearity is well perceived above $273.15 \mathrm{~K}\left(0^{\circ} \mathrm{C}\right)$ in Figure 7 with correlation coefficient 0.9995 , for which aqueous surfactant solutions are examined for micellization.

The linear relationship between $\Delta S_{m, c h}^{\prime \prime}$ and $T$ above $273.15 \mathrm{~K}$ can be shown by modifying eq. 21 . Eq. $2 \mathrm{I}$ can be rewritten as

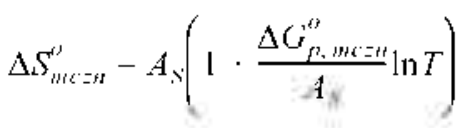

Let the reference temperature be $T_{0}(-273.15 \mathrm{~K})$ and $\delta=T_{-}$ 


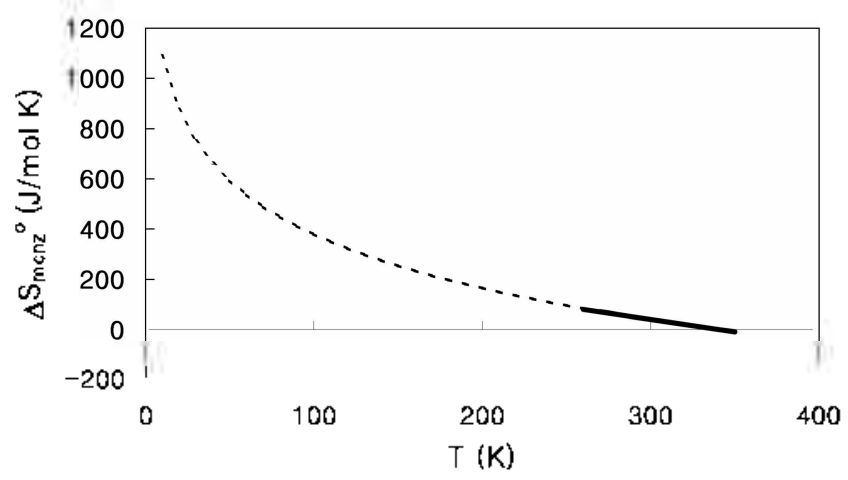

Figure $7, \Delta S_{m+2}^{\prime \prime}$ by eq. (21) and the portion at $\%>273 \mathrm{~K}$ for which $\Delta S_{m, n}$ changes linearly with temperature.

$T_{0 .}$ Then, this equation becomes

$$
\Delta S_{m c z n}^{o}=A_{S}\left[1+\frac{\Delta G_{p, m c z n}^{o}}{A_{S}}\left\{\ln T_{0}+\ln \left(1+\frac{\delta}{T_{0}}\right)\right\}\right]
$$

Txpanding into a power series yields

$$
\ln \left(1-\frac{\delta}{T_{0}}\right)=\frac{\delta}{T_{0}}-\frac{1}{2}\left(\frac{\delta}{T_{0}}\right)^{2}+\frac{1}{3}\left(\frac{\delta}{T_{0}}\right)^{3}-\cdots
$$

The first term only is maintained because $\delta / T_{0}$ is very small. Hence, $\Delta S_{m c=n}^{\prime \prime}$ can be well approximated as

$$
\begin{aligned}
& \Delta S_{m i=n}-A_{S}\left[1+\frac{\Delta G_{n, m \in n}^{n}}{A_{Y}}\left(\ln T_{0}+\frac{\delta}{T_{0}}\right)\right]
\end{aligned}
$$

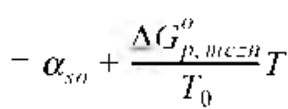

where $\alpha_{x,}$ is defined as

$$
\alpha_{\mathrm{s}, t}-A_{\mathrm{i}}+\Delta G_{p, m=m}^{\prime \prime}\left(\ln T_{0}-\mathrm{l}\right)
$$

Eq. 22 shows that $\Delta S_{m+z=}^{\prime \prime}$ changes linearly with temperature above $273.15 \mathrm{~K}$.

The corollary of the new cquation, eq. 20 , is that it accounts for the compensation phenomena, which states that large change in $\Delta H_{m t^{\prime \prime}, \prime}^{\prime \prime}$ and $\Delta S_{t \prime \prime \prime \prime \prime}^{\prime \prime}$ compensate each other, leaving $\Delta G_{m(z=)}^{t)}$ almost invariant and small in magnitude, and that $\Delta H_{m=t \prime}^{\prime \prime}$ changes linearly with $\Delta S_{m(z),}^{\prime \prime}$. The linear bchavior of $\Delta S_{m=t}^{\prime \prime}$ with temperature, cq. 22 , is resulted from eq. 20. Combining eq. 22 with eq. 13, observed linear behavior of $\Delta H_{m=t \prime}^{\prime \prime}$ with $T$, onc oblains

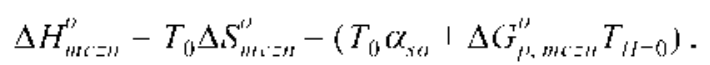

This equation clearly shows that $\Delta H_{m c^{\prime \prime}=}^{\prime \prime}$ changes linearly with $\Delta S_{m=n}^{\prime \prime}$ or vice versa. The reference temperature $T_{0}$ is called the compensation temperalure and characterizes the compensation phenomenon. $T_{0}$ has been lound to be $307 \perp 7$ $\mathrm{K}$ for 11 surfactants ${ }^{22}$ and this value may be in practice independent of surfactant systems. ${ }^{23}$ Figure 8 shows the linearity between $\Delta H_{m c^{\prime \prime},}^{\prime \prime}$ and $\Delta S_{m c^{\prime \prime \prime}}^{\prime \prime}$ whose values are

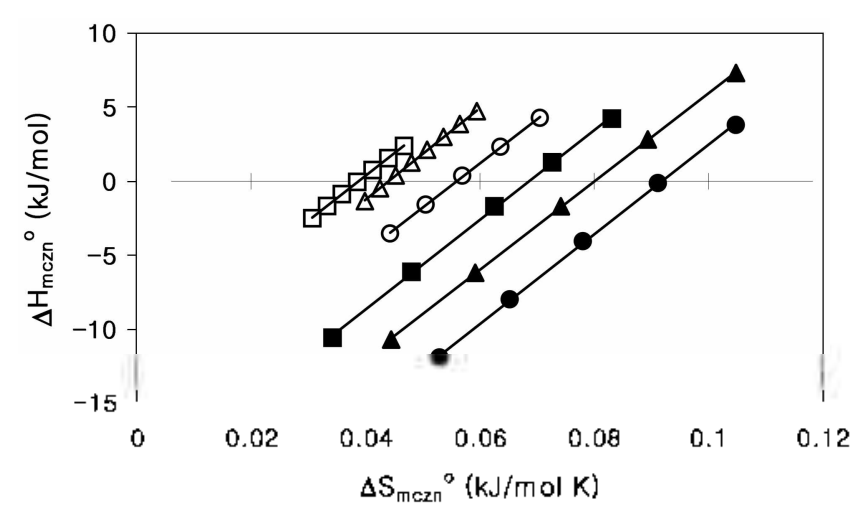

Figure 8. The linearity between $\Delta H_{m}^{\prime s}$ and $\Delta S_{m(z)}^{\prime \prime}$ for cationic surfactants $C^{\prime} I A B(\lrcorner: i=6$. ${ }^{\prime}: i=8 . \leq: i=10 . \square: i=12 . \diamond: i=14$. A : $i=16)$.

obtained from calculations by eqs. 15 and 22 for cationic surfactant $\left(C, T A B, i=6,^{32} 8,,^{67} 10,,^{3.7} 12,,^{3.1} 14, a^{35} 16^{36}\right)$ systems. Other types of surfactants also exhibit such behavior. ${ }^{.1}$ This result again manifests strongly that the new equation of $V_{\text {a }}(T)$ describes correctly the temperature dependence of $\mathrm{CMC}$.

\section{Conclusions}

A new equation, $\ln X_{C M}=A+B \ln T-(C / T)$ has been derived on the basis of $\Delta G^{\prime \prime}=-R T \ln K$, linear behavior of the enthalpy of micellization with temperature, and the Gibbs-Helmholtz. realtion. This equation describes the temperature dependence of critical micelle concentration (CMC), $X_{1: k}$, with three parameters, $A, B$, and $C$. When tested for surfactant systems of various kinds, the equation fits excellently the measured CMC of these surfactant systems. This result may imply that the new ecuation holds without regard to surfactant systems.

The corollary of the new equation is that the entropy of micellization, $\Delta S_{m c z h}^{\prime \prime}$, varies linearly with temperature. This result, when combined with the linear behavior of the entropy of micellization, $\Delta H_{H i c z}^{\prime \prime}$, with temperature, accounts for the compensation phenomena observed for the micellization in aqueous surfactant solutions.

Acknowledgements. H.-U. Kim was gratefully acknowledged to support of the Korea Research Foundation through the grant (No: EN0037).

\section{References}

1. Viller. D. D.: Magid. L. J.: Evans. D. F. . Plys (hem. 1990. 94. 5921.

2. Becher. P. In Nonionic Stufoctanis: Schick. M. I.. Iid.: Marcel Dekker: New York. 1967: Chapter 5.

3. Flockhart. B. D.J. Colloid hnterface Sci. 1961. 16. 484.

4. Stead. J. A.: Taylor. H. J. J. (olloid Interferes Sci. 1969. 30. 482.

5. Blandamer. M. 1.: Cullis. P. M.: Soldi. L. G.: Engberts. J. B. F. N.: Kacperska, A.: Van Os. N. M.: Subhis M. C. S. Adv Colloid Interface Ser. 1995. 58. 171 .

6. Shinoda. K.: Kobayalshi. M.: Yamaguchi. .. J. Phis. Chem. 1987. 
91, 5292

7. Okawauchi. M.: Gagio. M.: Ikawa. Y.: Sugihara. G.: Murata. Y: Tanaka. M. Bull. Chent. Soc. Jpn. 1987.60.2718.

8. Chen. L.-J.: Lin. S.-Y.: Huang. C.-C.: Chen. E-M. Colloid Sirf. A $1998,135,175$.

9. Zielinski. R. J. Colloid hnterface Sci. $2001,235.201$.

10. Kang. K.-H.: Kim. H.-U.; Lim. K.-H. Collord Surf. A 2001 . 189. 113.

11. Kresheck. G. C.: Hargraves. W. A. J. Colloid Interface Sci. 1974. 48.481 .

12. Tomasic, V.: Chittofrati. A.; Kallay, N. Colloid Surf A 1995, 104. 95 .

13. Kiraly. A.: Dekany. I. J. Colloid hiterface Sci. 2002. 242, 214.

14. Paula. S.: Sus. W.: Tuchtenhagen. J.: Blume. A. J. Phus. Chent. 1995. 99.11742

15. Muller. N. Langntuir 1993.9.96.

16. Singh. H. N.: Saleem. S. M.: Singli. R. P. J. Phys. (Them. 1980, 84. 2191.

17. Gilli. P: Ferretti, V: Gilli, G.: Borea, P. A. J. Ptrys. Chem 1993. 98.1515.

18. Madan. B.: Lee. B. Bioplws. Chent. 1994. 51.279.

19. Lumry. R.: Rajender. S. Biopolnmers 1970.9.1125.

20. Jolicoeur C.: Philip, R. P. Can. J. Chem. 1974, 52. 1834

21. Krishnan. V. C. Friedman. L. H. J. Solution (7hom. 1974. 2.37.

22. Sugihara. G.: Hisatomi. M. J. Colloid hiterface Sci. 1999, 219. 31 .

23. Kim. H.-U.: Lim. K.-H. manuscript in preparation. 2003

24. Tanford. C. Hydrophobic Effects: Formation of Hicelles and Biological Membrames: John Wiley and Sons. Inc: New York. 1980.
25. Phillips. J. N. Trans. Faraday Soc. 1955. 151, 561.

26. Kimn. H. U. Ph. D. Dissentation: Chung-Ang University: Seoul. Korea. 2003.

27. Kim. H.-U.: Lim. K.-H. submitted to Colloid Surf. A. 2003.

28. Moroi. Y. Aficelles: Theontical and Applied Aspects, Plenum New York, 1992.

29. Sugihara, G; Arakawa, Y.: Tanaka. K: Lee, S.: Moroi, Y. $J$. Colloid Interface Sei. 1995. 170. 399.

30. Muller. N. In Micellization. Solubilization, and Hichemulsions: Mittal. K. L.. Ed.: Plenum: New York. 1977: Vol. 1. pp 229-239

31. Holtzer. A.; Holtzer, M. F. J. Phys. Chem 1974, 78 , 1442.

32. Mosquera. V: del Rip, J. M.; Attwood, D: Garcia. M.: Jones. M. N.; Prieto. G.: Suarez. M. J.: Sarmiento, F. J. Colloid Interface Sci. 1998. 206.66.

33. Yoshida. N.: Matsuoka. K.: Moroi. Y. J. Colloid Interface Sci. 1997. 187. 388 .

34. La Mesa, C. J. Phus. Chem 1990, 94. 323.

35. Castedo. A.: Del Castillo. J. L.: Suarez-Filloy: M. J.: Rodriguez. J. R. J. Colloid hiterface Sci 1997, 196. 148.

36. Lee. D. J. Colloid Pohm. Sci. 1995. 273. 539.

37. Moroi. Y.: Nishikino. N.: Uehara. H.: Matuura. R. J. Colloid Interface Sci. 1975. 50.254.

38. Ruso, J. M; Taboada. P; Mosquera. V; Sarmiento. F. J. Colloid Interface Sci. 1999, 214. 292

39. Taboada, P; Attwood, D; Garcia, M.; Jones, M. N.: Ruso. J. M.: Mosquera. V: Sarmiento. F. J. Colloid Interface Sci. 2000.221. 242.

40. Attwood. D.: Boitard. E.: Dubes. T.-P.: Tachoire. H. J. Colloid Interface Sci. $\mathbf{2 0 0 0}, 227.356$.

41. Kim. H.-U.: Lim. K.-H. manuscript in preparation. 2003. 